\title{
Nonlinear vibrations of piles in viscoelastic foundations
}

\author{
E. J. Sapountzakis \& A. E. Kampitsis \\ School of Civil Engineering, \\ National Technical University of Athens, Greece
}

\begin{abstract}
In this paper, a boundary element method is developed for the nonlinear dynamic analysis of piles of arbitrary doubly symmetric simply or multiply connected constant cross section, partially embedded in viscoelastic foundations, undergoing moderate large deflections under general boundary conditions, taking into account the effects of shear deformation and rotary inertia. The pile is subjected to the combined action of arbitrarily distributed or concentrated transverse loading and bending moments in both directions as well as to axial loading. To account for shear deformations, the concept of shear deformation coefficients is used. Five boundary value problems are formulated with respect to the transverse displacements, to the axial displacement and to two stress functions and solved using the Analog Equation Method, a BEM based method. Application of the boundary element technique yields a nonlinear coupled system of equations of motion. The solution of this system is accomplished iteratively by employing the average acceleration method in combination with the modified Newton Raphson method. The evaluation of the shear deformation coefficients is accomplished from the aforementioned stress functions using only boundary integration. The proposed model takes into account the coupling effects of bending and shear deformations along the member as well as the shear forces along the span induced by the applied axial loading. Numerical examples are worked out to illustrate the efficiency, wherever possible the accuracy and the range of applications of the developed method.

Keywords: piles, nonlinear vibrations, large deflections, Timoshenko beam, shear deformation coefficients, boundary element method, viscoelastic foundation.
\end{abstract}




\section{Introduction}

Many problems related to soil-structure interaction can be modelled by means of a beam or a beam-column on an elastic foundation. Practical examples of these are railroad tracks, highway pavements, continuously supported pipelines, and strip foundations. Moreover, piles are frequently employed for the foundation of structures such as buildings, quay walls, bridges and offshore structures. These piles, which are subjected to lateral forces that result from loading on supported structures, during earthquake excitation, develop a nonlinear dynamic response. Thus, the study of nonlinear effects on the dynamic analysis of structural elements is essential in civil engineering applications, wherein weight saving is of paramount importance. This non-linearity results from retaining the square of the slope in the strain-displacement relations (intermediate non-linear theory), avoiding in this way the inaccuracies arising from a linearized second-order analysis. Thus, the aforementioned study takes into account the influence of the action of axial, lateral forces and end moments on the deformed shape of the structural element. Moreover, due to the intensive use of materials having relatively high transverse shear modulus and the need for beam members with high natural frequencies the error incurred from the ignorance of the effect of shear deformation may be substantial, particularly in the case of heavy lateral loading. The Timoshenko-Rayleigh beam theory, which includes shear deformation and rotary inertia effects has an extended range of applications as it allows treatment of deep beam (depth is large relative to length), short and thinwebbed beams and beams where higher modes are excited.

When the beam-column deflections of the structure are small, a wide range of linear analysis tools, such as modal analysis, can be used, and some analytical results are possible. During the past few years, the linear dynamic analysis of beams on elastic foundation has received a good amount of attention in the literature with pioneer the work of Hetenyi [1] who studied the elementary Bernoulli-Euler beams on elastic Winkler foundation. Rades [2] presented the steady-state response of a finite rigid beam resting on a foundation defined by one inertial and three elastic parameters in the assumption of a permanent and smooth contact between beam and foundation considering only uncoupled modes. Wang and Stephens [3] studied the natural vibrations of a Timoshenko beam on a Pasternak-type foundation showing the effects of rotary inertia, shear deformation and foundation constants of the beam employing general analytic solutions for simple cases of boundary conditions. De Rosa [4] and El-Mously [5] derived explicit formulae for the fundamental natural frequencies of finite Timoshenko-beams mounted on finite Pasternak foundation.

Moreover, El Naggar and Novak [6] studied the lateral response of singe piles and pile groups accounting the nonlinear behaviour of the soil adjacent to the pile and discontinuity conditions at the pile-soil interface. Padron et. al. [7] studied a BEM-FEM coupling model for the time harmonic dynamic analysis of piles and pile groups embedded in an elastic half-space where piles are modelled using finite elements as a beam according to the Bernoulli hypothesis, while the soil is modelled using boundary elements as a continuum, semi-infinite, 
isotropic, homogeneous or zoned homogeneous, linear, viscoelastic medium. Hu et al. [8] presented the nonlinear partial differential equation governing the nonlinear transverse vibration of pile under the assumption that both the materials of the pile and the soil obey nonlinear elastic and linear viscoelastic constitutive relations while the frequency and the response of the system have been obtained by the complex mode method and the method of multiple time scales.

As the deflections become larger, the induced geometric nonlinearities result in effects that are not observed in linear systems. Contrary to the good amount of attention in the literature concerning the linear dynamic analysis of beamcolumns supported on elastic foundation, little work has been done on the corresponding nonlinear problem, such as the nonlinear free vibration analysis of multispan beams on elastic supports presented by Lewandowski [9], employing the dynamic finite element method, neglecting the horizontally and rotary inertia forces and considering the beams as distributed mass systems.

In this paper, a boundary element method is developed for the nonlinear dynamic analysis of piles of arbitrary doubly symmetric simply or multiply connected constant cross section, partially embedded in viscoelastic foundation, undergoing moderate large deflections under general boundary conditions, taking into account the effects of shear deformation and rotary inertia. The pile is subjected to the combined action of arbitrarily distributed or concentrated transverse loading and bending moments in both directions as well as to axial loading. To account for shear deformations, the concept of shear deformation coefficients is used. Five boundary value problems are formulated with respect to the transverse displacements, to the axial displacement and to two stress functions and solved using the Analog Equation Method [10], a BEM based method. Application of the boundary element technique yields a nonlinear coupled system of equations of motion. The solution of this system is accomplished iteratively by employing the average acceleration method in combination with the modified Newton Raphson method [11, 12]. The evaluation of the shear deformation coefficients is accomplished from the aforementioned stress functions using only boundary integration. The essential features and novel aspects of the present formulation compared with previous ones are summarized as follows.

i. Shear deformation effect and rotary inertia are taken into account on the nonlinear dynamic analysis of piles subjected to arbitrary loading (distributed or concentrated transverse loading and bending moments in both directions, as well as axial loading).

ii. The homogeneous linear half-space is approximated by a viscoelastic foundation.

iii. The pile is supported by the most general nonlinear boundary conditions including elastic support or restrain, while its cross section is an arbitrary doubly symmetric one.

iv. The proposed model takes into account the coupling effects of bending and shear deformations along the member as well as shear forces along the span induced by the applied axial loading. 
v. The shear deformation coefficients are evaluated using an energy approach, instead of Timoshenko and Goodier's [13] and Cowper's [14].

vi. The effect of the material's Poisson ratio $v$ is taken into account.

vii. The proposed method employs a BEM approach (requiring boundary discretization) resulting in line or parabolic elements instead of area elements of the FEM solutions (requiring the whole cross section to be discretized into triangular or quadrilateral area elements), while a small number of line elements are required to achieve high accuracy.

\section{Statement of the problem}

Let us consider a prismatic pile of length $l$, of constant arbitrary doubly symmetric cross-section of area $A$. The homogeneous isotropic and linearly elastic material of the pile cross-section, with modulus of elasticity $E$, shear modulus $G$ and Poisson's ratio $v$ occupies the two dimensional multiply connected region $\Omega$ of the $y, z$ plane and is bounded by the $\Gamma_{j}(j=1,2, \ldots, K)$ boundary curves, which are piecewise smooth, i.e. they may have a finite number of corners. Consider $C y z$ to be the principal bending coordinate system through the cross section's centroid. The pile is partially embedded in a homogeneous viscoelastic soil. The foundation model is characterized by the Winkler moduli $k_{y}, k_{z}$ and the damping coefficients $c_{y}, c_{z}$ corresponding to the directions $y, z$ respectively. Thus, the foundation reaction is written as

$$
\begin{aligned}
& p_{s y}(x, t)=k_{y} v(x, t)+c_{y} \frac{\partial v(x, t)}{\partial t} \\
& p_{s z}(x, t)=k_{z} w(x, t)+c_{z} \frac{\partial w(x, t)}{\partial t}
\end{aligned}
$$

The pile is subjected to the combined action of the arbitrarily distributed or concentrated time dependent axial loading $p_{x}=p_{x}(x, t)$, transverse loading $p_{y}=p_{y}(x, t), p_{z}=p_{z}(x, t)$ acting in the $y, z$ directions, respectively and bending moments $m_{y}=m_{y}(x, t), m_{z}=m_{z}(x, t)$ along $y, z$ axes, respectively.

Under the action of the aforementioned loading, the displacement field of the pile taking into account shear deformation effect is given as

$$
\begin{aligned}
\bar{u}(x, y, z, t)= & u(x, t)-y \theta_{z}(x, t)+z \theta_{y}(x, t) \\
& \bar{v}(x, t)=v(x, t) \\
& \bar{w}(x, t)=w(x, t)
\end{aligned}
$$

where $\bar{u}, \bar{v}, \bar{w}$ are the axial and transverse pile displacement components with respect to the $C y z$ system of axes; $u(x, t), v(x, t), w(x, t)$ are the 
corresponding components of the centroid $C$ and $\theta_{y}(x, t), \theta_{z}(x, t)$ are the angles of rotation due to bending of the cross-section with respect to its centroid.

Employing the strain-displacement relations of the three-dimensional elasticity for moderate displacements, the following strain components can be easily obtained

$$
\begin{gathered}
\varepsilon_{x x}=\frac{\partial \bar{u}}{\partial x}+\frac{1}{2}\left[\left(\frac{\partial \bar{v}}{\partial x}\right)^{2}+\left(\frac{\partial \bar{w}}{\partial x}\right)^{2}\right] \\
\gamma_{x z}=\frac{\partial \bar{w}}{\partial x}+\frac{\partial \bar{u}}{\partial z}+\left(\frac{\partial \bar{v}}{\partial x} \frac{\partial \bar{v}}{\partial z}+\frac{\partial \bar{w}}{\partial x} \frac{\partial \bar{w}}{\partial z}\right) \\
\gamma_{x y}=\frac{\partial \bar{v}}{\partial x}+\frac{\partial \bar{u}}{\partial y}+\left(\frac{\partial \bar{v}}{\partial x} \frac{\partial \bar{v}}{\partial y}+\frac{\partial \bar{w}}{\partial x} \frac{\partial \bar{w}}{\partial y}\right) \\
\varepsilon_{y y}=\varepsilon_{z z}=\gamma_{y z}=0
\end{gathered}
$$

where it has been assumed that for moderate displacements $(\partial \bar{u} / \partial x)^{2}<<\partial \bar{u} / \partial x$, $(\partial \bar{u} / \partial x)(\partial \bar{u} / \partial z)<(\partial \bar{u} / \partial x)+(\partial \bar{u} / \partial z), \quad(\partial \bar{u} / \partial x)(\partial \bar{u} / \partial y)<<(\partial \bar{u} / \partial x)+(\partial \bar{u} / \partial y)$.

Substituting the displacement components to the strain-displacement relations, the strain components can be written as

$$
\begin{gathered}
\varepsilon_{x x}(x, y, z, t)=u^{\prime}+z \theta_{y}{ }^{\prime}-y \theta_{z}{ }^{\prime}+\frac{1}{2}\left(v^{\prime 2}+w^{\prime 2}\right) \\
\gamma_{x y}=v^{\prime}-\theta_{z} \\
\gamma_{x z}=w^{\prime}+\theta_{y}
\end{gathered}
$$

where $\gamma_{x y}, \gamma_{x z}$ are the additional angles of rotation of the cross-section due to shear deformation .

Considering strains to be small, employing the second Piola - Kirchhoff stress tensor and assuming an isotropic and homogeneous material, the stress components are defined in terms of the displacement ones as

$$
\begin{gathered}
S_{x x}=E\left[u^{\prime}+z \theta_{y}^{\prime}-y \theta_{z}^{\prime}+\frac{1}{2}\left(v^{\prime 2}+w^{\prime 2}\right)\right] \\
S_{x y}=G \cdot\left(v^{\prime}-\theta_{z}\right) \\
S_{x z}=G \cdot\left(w^{\prime}+\theta_{y}\right)
\end{gathered}
$$

On the basis of Hamilton's principle, the variations of the Lagrangian equation defined as

$$
\delta \int_{t_{1}}^{t_{2}}\left(U-K-W_{e x t}\right) d t=0
$$


and expressed as a function of the stress resultants acting on the cross section of the pile in the deformed state provide the governing equations and the boundary conditions of the pile subjected to nonlinear vibrations. In eqn. (16), $\delta(\cdot)$ denotes variation of quantities while $U, K, W_{\text {ext }}$ are the strain energy, the kinetic energy and the external load work. Moreover, the stress resultants of the pile using the expressions of the stress components are given as

$$
\begin{gathered}
N=E A\left[u^{\prime}+\frac{1}{2}\left(v^{\prime 2}+w^{\prime 2}\right)\right] \\
M_{y}=E I_{y} \theta_{y}^{\prime} \\
M_{z}=E I_{z} \theta_{z}^{\prime} \\
Q_{y}=G A_{y} \gamma_{x y} \\
Q_{z}=G A_{z} \gamma_{x z}
\end{gathered}
$$

where $A$ is the cross section area, $I_{y}, I_{z}$ the moments of inertia with respect to the principle bending axes and $G A_{y}, G A_{z}$ are its shear rigidities of the Timoshenko's beam theory, where

$$
A_{z}=\kappa_{z} A=\frac{1}{a_{z}} A \quad A_{y}=\kappa_{y} A=\frac{1}{a_{y}} A
$$

are the shear areas with respect to $y, z$ axes, respectively with $\kappa_{y}, \kappa_{z}$ the shear correction factors and $a_{y}, a_{z}$ the shear deformation coefficients. Substituting the stress components and the strain resultants to the strain energy variation and employing eqn. (16), the equilibrium equations of the pile are derived as

$$
\begin{gathered}
-E A\left(u^{\prime \prime}+w^{\prime} w^{\prime \prime}+v^{\prime} v^{\prime \prime}\right)+\rho A \ddot{u}=p_{x} \\
E I_{z} v^{\prime \prime \prime \prime}+\rho A \ddot{v}+p_{s y}+\frac{E I_{z}}{G A_{y}}\left(\left(N v^{\prime}\right)^{\prime \prime \prime}-\rho A \frac{\partial^{2} \ddot{v}}{\partial x^{2}}-p_{s y}{ }^{\prime \prime}+p_{y} \prime\right)-\left(N v^{\prime}\right)^{\prime} \\
-\rho I_{z} \frac{\partial^{2} \ddot{v}}{\partial x^{2}}-\frac{\rho I_{z}}{G A_{y}}\left(\frac{\partial^{2}\left(N v^{\prime}\right)^{\prime}}{\partial t^{2}}-\rho A \dddot{v}-\ddot{p}_{s y}+\ddot{p}_{y}\right)=p_{y}-m_{z}^{\prime} \\
E I_{y} w^{\prime \prime \prime \prime}+\rho A \ddot{w}+p_{s z}+\frac{E I_{y}}{G A_{z}}\left(\left(N w^{\prime}\right)^{\prime \prime \prime}-\rho A \frac{\partial^{2} \ddot{w}}{\partial x^{2}}-p_{s z}{ }^{\prime \prime}+p_{z}^{\prime \prime}\right)-\left(N w^{\prime}\right)^{\prime} \\
-\rho I_{z} \frac{\partial^{2} \ddot{w}}{\partial x^{2}}-\frac{\rho I_{y}}{G A_{z}}\left(\frac{\partial^{2}\left(N w^{\prime}\right)^{\prime}}{\partial t^{2}}-\rho A \dddot{w}-\ddot{p}_{s z}+\ddot{p}_{z}\right)=p_{z}+m_{y}^{\prime}
\end{gathered}
$$


Eqns. (23)-(25) constitute the governing differential equations of a Timoshenko-Rayleigh pile, partially embedded in viscoelastic foundation, subjected to nonlinear vibrations due to the combined action of time dependent axial and transverse loading. These equations are also subjected to the pertinent boundary conditions of the problem, which are given as

$$
\begin{gathered}
a_{1} u(x, t)+\alpha_{2} N(x, t)=\alpha_{3} \\
\beta_{1} v(x, t)+\beta_{2} V_{y}(x, t)=\beta_{3} \quad \bar{\beta}_{1} \theta_{z}(x, t)+\bar{\beta}_{2} M_{z}(x, t)=\bar{\beta}_{3} \\
\gamma_{1} w(x, t)+\gamma_{2} V_{z}(x, t)=\gamma_{3} \quad \bar{\gamma}_{1} \theta_{y}(x, t)+\bar{\gamma}_{2} M_{y}(x, t)=\bar{\gamma}_{3}
\end{gathered}
$$

at the pile ends $x=0, l$, together with the initial conditions

$$
\begin{array}{rr}
u(x, 0)=\bar{u}_{0}(x) & \dot{u}(x, 0)=\dot{\bar{u}}_{0}(x) \\
v(x, 0)=\bar{v}_{0}(x) & \dot{v}(x, 0)=\dot{\bar{v}}_{0}(x) \\
w(x, 0)=\bar{w}_{0}(x) & \dot{w}(x, 0)=\dot{\bar{w}}_{0}(x)
\end{array}
$$

where $\bar{u}_{0}(x), \bar{v}_{0}(x), \bar{w}_{0}(x), \dot{\bar{u}}_{0}(x), \dot{\bar{v}}_{0}(x)$ and $\quad \dot{\bar{w}}_{0}(x)$ are prescribed functions. In eqns. (27), (28) $V_{y}, V_{z}, M_{z}, M_{y}$ and $\theta_{y}, \theta_{z}$ are the reactions, the bending moments and the angles of rotation due to bending with respect to $y$, $z$, respectively.

Finally, $\alpha_{k}, \beta_{k}, \bar{\beta}_{k}, \gamma_{k}, \bar{\gamma}_{k}(k=1,2,3)$ are functions specified at the pile ends $x=0, l$. Eqs. (26)-(28) describe the most general nonlinear boundary conditions associated with the problem at hand and can include elastic support or restraint. It is apparent that all types of the conventional boundary conditions (clamped, simply supported, free or guided edge) can be derived from these equations by specifying appropriately these functions (e.g. for a clamped edge it is $\alpha_{2}=\alpha_{3}=$ $\left.\beta_{2}=\beta_{3}=\gamma_{2}=\gamma_{3}=\bar{\beta}_{2}=\bar{\beta}_{3}=\bar{\gamma}_{2}=\bar{\gamma}_{3}=0, \alpha_{1}=\beta_{1}=\gamma_{1}=1, \bar{\beta}_{1}=\bar{\gamma}_{1}=1\right)$.

The solution of the initial boundary value problem given from eqns. (23)(25), subjected to the boundary conditions (26)-(28) and the initial conditions (29)-(31) which represents the nonlinear flexural dynamic analysis of a Timoshenko-Rayleigh pile, partially embedded in viscoelastic foundation, presumes the evaluation of the shear deformation coefficients $a_{y}, a_{z}$, corresponding to the principal coordinate system $C y z$. These coefficients are established equating the approximate formula of the shear strain energy per unit 
length [15] $U_{\text {appr. }}=\frac{a_{y} Q_{y}^{2}}{2 A G}+\frac{a_{z} Q_{z}^{2}}{2 A G}$ with the exact one given from $U_{\text {exact }}=\int_{\Omega} \frac{\left(\tau_{x z}\right)^{2}+\left(\tau_{x y}\right)^{2}}{2 G} d \Omega$ and are obtained as [16]

$$
\begin{aligned}
& a_{y}=\frac{1}{\kappa_{y}}=\frac{A}{\Delta^{2}} \int_{\Omega}[(\nabla \Theta)-\boldsymbol{e}] \cdot[(\nabla \Theta)-\boldsymbol{e}] d \Omega \\
& a_{z}=\frac{1}{\kappa_{z}}=\frac{A}{\Delta^{2}} \int_{\Omega}[(\nabla \Phi)-\boldsymbol{d}] \cdot[(\nabla \Phi)-\boldsymbol{d}] d \Omega
\end{aligned}
$$

where $\left(\tau_{x z}\right)_{j},\left(\tau_{x y}\right)_{j}$ are the transverse (direct) shear stress components, $(\nabla) \equiv \boldsymbol{i}_{\boldsymbol{y}}(\partial / \partial y)+\boldsymbol{i}_{\boldsymbol{z}}(\partial / \partial z)$ is a symbolic vector with $\boldsymbol{i}_{\boldsymbol{y}}, \boldsymbol{i}_{\boldsymbol{z}}$ the unit vectors along $y$ and $z$ axes, respectively. Moreover, $\Delta=2(1+v) I_{y} I_{z}$ where $v$ is the Poisson ratio of the cross section material, $\boldsymbol{e}=\left(v I_{y} \frac{y^{2}-z^{2}}{2}\right) \boldsymbol{i}_{\boldsymbol{y}}+v I_{y} y z \boldsymbol{i}_{z}$ and $\boldsymbol{d}=v I_{z} y z \boldsymbol{i}_{\boldsymbol{y}}-\left(v I_{z} \frac{y^{2}-z^{2}}{2}\right) \boldsymbol{i}_{z}$ while $\Theta(y, z)$ and $\Phi(y, z)$ are stress functions which are evaluated from the solution of the following Neumann type boundary value problems [16]

$$
\begin{gathered}
\nabla^{2} \Theta=-2 I_{y} y \quad \text { in } \Omega \\
\frac{\partial \Theta}{\partial n}=\boldsymbol{n} \cdot \boldsymbol{e} \text { on } \Gamma=\bigcup_{j=1}^{K+1} \Gamma_{j} \\
\nabla^{2} \Phi=-2 I_{z} z \quad \text { in } \Omega \\
\frac{\partial \Phi}{\partial n}=\boldsymbol{n} \cdot \boldsymbol{d} \text { on } \Gamma=\bigcup_{j=1}^{K+1} \Gamma_{j}
\end{gathered}
$$

where $\boldsymbol{n}$ is the outward normal vector to the boundary $\Gamma$. In the case of negligible shear deformations $a_{z}=a_{y}=0$. It is also worth here noting that the boundary conditions (37), (39) have been derived from the physical consideration that the traction vector in the direction of the normal vector $n$ vanishes on the free surface of the pile. 


\section{Integral representations - numerical solution}

According to the precedent analysis, the nonlinear flexural dynamic analysis of Timoshenko-Rayleigh pile, partially embedded in viscoelastic foundation, undergoing moderate large deflections reduces in establishing the displacement components $u(x, t)$ and $v(x, t), w(x, t)$ having continuous derivatives up to the second order and up to the fourth order with respect to $x$, respectively, and also having derivatives up to the second order with respect to $t$ (ignoring the inertia terms of the fourth order [17]). These displacement components must satisfy the coupled governing differential eqns. (23)-(25) inside the pile, the boundary conditions (26)-(28) at the pile ends $x=0, l$ and the initial conditions (29)-(31). Eqns. (23)-(25) are solved using the Analog Equation Method [10] as it is developed for hyperbolic differential equations [18].

\section{Numerical examples}

On the basis of the analytical and numerical procedures presented, a computer program has been written and a representative example has been studied to demonstrate the efficiency of the developed method. In this example, the results have been obtained using $L=41$ nodal points along the pile and a time step of $\Delta t=1.0 \mu \mathrm{sec}$.

\subsection{Example}

A partially embedded pile of total length $l=10 \mathrm{~m}\left(l_{\text {free }}=3.0 \mathrm{~m}, l_{\text {embed }}=7 \mathrm{~m}\right)$, of circular cross section of diameter $D=0.5 \mathrm{~m}$ ( $E=29 \mathrm{GPa}, \quad v=0.2$, $\left.A=0.196 \mathrm{~m}^{2}, I_{y}=I_{z}=3.066 \cdot 10^{-3} \mathrm{~m}^{4}\right)$ is studied. The foundation model is characterized by the Winkler modulus $k=17.4 \mathrm{MN} / \mathrm{m}^{2}$ and the damping coefficient $c=12 \mathrm{kNs} / \mathrm{m}^{2}$. According to its boundary conditions, the embedded pile end is free, while the other end is free according to its displacements and blocked according to its rotations. The pile is subjected to a concentrated compressive axial load $P_{x}(0, t)=1500 \mathrm{kN},(t \geq 0.0)$ and to a concentrated transverse force $P_{z}(0, t)=1000 \mathrm{kN},(t \geq 0.0)$ acting at its top.

In fig. 1 the time histories of the head displacement $w_{t o p}(0, t)$ of the pile embedded in a viscoelastic foundation are presented taking into account the rotary inertia and the shear deformation effect, for two values of the damping coefficient ( $\left.c=0 \mathrm{kNs} / \mathrm{m}^{2}, c=12 \mathrm{kNs} / \mathrm{m}^{2}\right)$ and performing either a linear or a nonlinear analysis. Moreover, in table 1 the maximum values of the head displacement $\left(w_{t o p}\right)_{\max }$ and the periods $T_{z}$ of the first-cycle of motion are presented for the aforementioned viscous cases of analysis. 

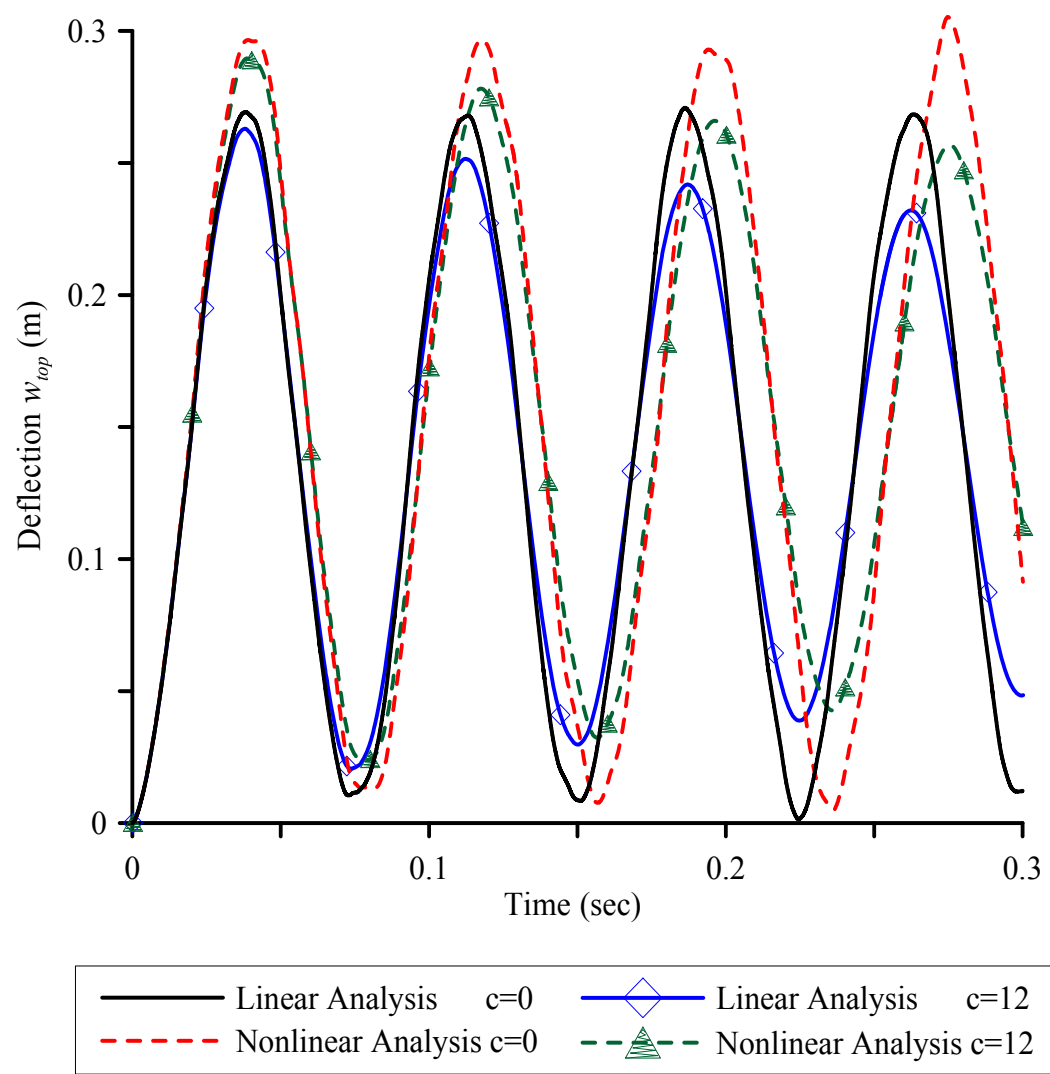

Figure 1: Time history of the transverse displacement $w_{t o p}(0, t)$ of the pile head.

Table 1: Maximum head displacement $\left(w_{t o p} \times 10^{-2} \mathrm{~m}\right)_{\max }$ and period $T_{z}\left(10^{-2} \mathrm{sec}\right)$ of the first cycle of motion of the pile.

\begin{tabular}{|c|c|c|c|}
\hline \multicolumn{2}{|c|}{ Nonlinear Analysis } & \multicolumn{2}{|c|}{ Linear Analysis } \\
\hline$\left(w_{\text {top }}\right)_{\max }$ & $T_{z}$ & $\left(w_{\text {top }}\right)_{\max }$ & $T_{z}$ \\
\hline 29.662 & 8.10 & 26.956 & 7.28 \\
\hline
\end{tabular}

Finally, in order to demonstrate the coupling effect of the transverse displacements in both directions in the nonlinear analysis, as a variant of the above application, the examined pile additionally to the already described 
loading is also subjected to a concentrated transverse force $P_{y}(0, t)=2000 \mathrm{kN}$, acting also at its top. In table 2 the maximum values of the head transverse displacements $\left(w_{\text {top }}\right)_{\max },\left(v_{\text {top }}\right)_{\max }$ are presented performing either a linear or a nonlinear analysis. The difference in the elements of the first columns of tables 1,2 is due to the coupling effect of the transverse displacements.

Table 2: Maximum head transverse displacements $\left(w_{\text {top }}\right)_{\max },\left(v_{t o p}\right)_{\max }$ $\left(\times 10^{-2}\right) m$ of the pile.

\begin{tabular}{|c|c|c|c|}
\hline \multicolumn{2}{|c|}{ Nonlinear Analysis } & \multicolumn{2}{|c|}{ Linear Analysis } \\
\hline$\left(w_{\text {top }}\right)_{\max }$ & $\left(v_{\text {top }}\right)_{\max }$ & $\left(w_{\text {top }}\right)_{\max }$ & $\left(v_{\text {top }}\right)_{\max }$ \\
\hline 29.671 & 59.343 & 26.956 & 53.912 \\
\hline
\end{tabular}

\section{Concluding remarks}

The main conclusions that can be drawn from this investigation are

a. The numerical technique presented in this investigation is well suited for computer aided analysis for piles of arbitrary simply or multiply connected doubly symmetric cross section.

b. The proposed method is developed for general dynamic analysis, while the pile is subjected to the most general boundary conditions and is embedded in viscoelastic foundation.

c. In some cases, the effect of shear deformation is significant, increasing the transverse displacements and decreasing the bending moments in both linear and nonlinear analysis.

d. The discrepancy between the results of the linear and the nonlinear analysis is remarkable.

e. The damping coefficient is of paramount importance for piles in viscoelastic foundations, as it reduces the vibration amplitude and the consequences of the dynamic response.

\section{Acknowledgements}

The work of this paper was conducted from the "DARE" project, financially supported by a European Research Council (ERC) Advanced Grant under the "Ideas" Programme in Support of Frontier Research [Grant Agreement 228254]. 


\section{References}

[1] Hetenyi, M., Beams and plates on elastic foundations and related problems, Applied Mechanics Reviews, 19, pp. 95-102, 1966.

[2] Rades, M., Dynamic analysis of an inertial foundation model, International Journal Solids Structures, 8, pp. 1353-1372, 1972.

[3] Wang, T.M. \& Stephens, J. E., Natural frequencies of Timoshenko beams on Pasternak foundation. Journal of Sound and Vibration, 51(2), pp. 149$155,1977$.

[4] De Rosa, M.A., Free vibrations of Timoshenko beams on two-parameter elastic foundation, Computers \& Structures, 57(1). pp. 151-156, 1995.

[5] El-Mously, M., Fundamental frequencies of Timoshenko beams mounted on Pasternak foundation, Journal of Sound and Vibration, 228(2), pp. 452-457, 1999.

[6] El Naggar, M.H. \& Novak, M., Nonlinear analysis for dynamic lateral pile response, Soil Dynamics and Earthquake Engineering, 15, pp. 233244, 1996.

[7] Padron, L.A., Aznarez, J.J. \& Maeso, O., BEM-FEM coupling model for the dynamic analysis of piles and pile groups, Engineering Analysis with Boundary Elements, 31, pp. 473-484, 2007.

[8] Hu, C.L., Cheng, C.J. \&Chen, Z.X., Nonlinear transverse free vibrations of piles, Journal of Sound and Vibration, 317, pp. 937-954, 2008.

[9] Lewandowski, R., Nonlinear free vibrations of multispan beams on elastic supports, Computers \& Structures, 32(2), pp. 305-312, 1989.

[10] Katsikadelis, J.T., The analog equation method. A boundary-only integral equation method for nonlinear static and dynamic problems in general bodies, Theoretical and Applied Mechanics, 27, pp. 13-38, 2002.

[11] Chang, S. Y. Studies of Newmark method for solving nonlinear systems: (I) basic analysis, Journal of the Chinese Institute of Engineers, 27(5), pp. 651-662, 2004.

[12] Isaacson, E. \& Keller, H.B., Analysis of Numerical Methods, John Wiley and Sons: New York: 1966.

[13] Timoshenko, S.P. \& Goodier, J.N., Theory of Elasticity, 3rd edn: McGraw-Hill: New York, 1984.

[14] Cowper, G.R., the shear coefficient in Timoshenko's beam theory. Journal of Applied Mechanics, ASME, 33(2), pp. 335-340, 1966.

[15] Stephen, N.G., Timoshenko's shear coefficient from a beam subjected to gravity loading, ASME Journal of Applied Mechanics, 47, pp. 121-127, 1980.

[16] Sapountzakis, E.J. \& Mokos, V.G., A BEM solution to transverse shear loading of beams, Computational Mechanics, 36, pp. 384-397, 2005.

[17] Thomson, W. T., Theory of vibration with applications, Englewood Cliffs Prentice Hall, 1981.

[18] Sapountzakis E.J. \& Katsikadelis J.T., Elastic deformation of ribbed plates under static, transverse and inplane loading, Computers and Structures, 74, pp. 571-581, 2000. 